\title{
Where am I looking? The accuracy of video-mediated gaze awareness
}

\author{
CAROLINE GALE and ANDREW F. MONK \\ University of York, York, England
}

\begin{abstract}
Participants worked in pairs, with one person gazing at a flat horizontal stimulus between them. The other participant estimated where the gazer was looking. Experiment 1 used linear scales as gaze targets. The mean root mean square error of estimation equates to $3.8^{\circ}$ of head-and-eye pan and $2.6^{\circ}$ of tilt. This small error of estimation was essentially the same in a video-mediated condition and in one in which a procedure that did not allow the estimator to see the head-and-eye movement to the target position was used. Experiment 2 obtained comparable gaze estimation performance in face-to-face and video-mediated conditions, using a combined pan-and-tilt grid. It is concluded that people are very good at estimating what someone else is looking at and that such estimations should be practical during video-mediated conversation.
\end{abstract}

How well are people able to judge where another person is looking? Can they do this over a video link? The reasons for asking these questions are essentially practical. Knowing where someone else is looking, or gaze awareness, as it will be termed here, might be expected to be a useful resource when communicating over a video link or in everyday face-to-face communication. Clark (1996) describes how head-and-eye movements are used to indicate references in conversation (e.g., "I want you [gazes at person A] and you [gazes at person B] to come with me."). Another, perhaps more controversial possibility is that we examine where people are looking when we talk to them as a way of knowing how well they have understood what was said.

Both of these possibilities depend on knowledge of what object in the environment someone is looking at. We define this as full gaze awareness and contrast it with partial gaze awareness, knowledge of only the general direction someone is looking in. Finally, one may distinguish a third class of gaze awareness, knowledge of whether someone is looking at you. Under normal conditions, it is only possible to know that someone is looking at your face if you are looking at theirs. For this reason, this kind of gaze awareness is known as mutual gaze, or eye contact. Whether or not it is possible to achieve these three kinds of gaze awareness over a video link depends very much on the view presented. For example, compare the head-and-shoulders view conventionally pre-

During this work, the first author was supported by a Research Studentship from the U.K. Engineering and Physical Science Research Council, the second by the U.K. Economic and Social Research Council through their Cognitive Engineering Programme. We thank Leon Watts for his contribution to setting up these experiments and his comments on drafts of this article. C.G. is now at BT Laboratories, Ipswich, England. Correspondence concerning this article should be addressed to A. Monk, Department of Psychology, University of York, York YO1 5DD, England (e-mail: a.monk@psych.york.ac.uk). sented in many desktop video conferencing applications with a view of the whole top half of the body and some of the surrounding room. In the latter case, one could potentially judge what someone is looking at (full gaze awareness) because both the viewer and the viewed object are visible. In the former case, this is not possible; one can only judge whether the viewer is looking up, down, left, or right (partial gaze awareness).

Most previous studies in which the role of gaze in communication has been examined have focused on mutual gaze (e.g., Goodwin, 1981; Kendon, 1967). It is technically difficult to provide realistic mutual gaze in video-mediated communication. This is because of the discrepancy between the position of the camera and the position of the face on the monitor. The simplest arrangement, again commonly used in commercial desktop video conferencing systems, is to place a camera on top of the monitor. If the viewer of one of these images looks directly at the image of the eyes of the other person on the screen, they are looking some way below the camera and so will appear to the person at the other end of the link to be looking somewhere on their abdomen. If they look directly at the camera they will appear to be making eye contact, but then they will not be able to see the face of the other person. To circumvent this problem, investigators at Xerox came up with the idea of a video tunnel (Buxton \& Moran, 1990). This uses half-silvered mirrors to put the camera in the same virtual position as the monitor. Surprisingly, there is no evidence that this ingenious invention leads to more effective conversations; indeed, it may have the opposite effect (Anderson et al., 1997).

The experiments reported here are not concerned with mutual gaze; rather, they focus on full gaze awareness. This follows a move, suggested by various authors, toward using video to show pictures of some object of shared work, rather than just pictures of the person one is talking to (Gaver, Smets, \& Overbeeke, 1995; Nardi et al., 1993; Siegel, Kraut, \& Miller, 1996; Watts \& Monk, 1996). If 
the purpose of a video link is to provide information about the work being done, rather than just facial expressions, it becomes important to determine whether full gaze awareness can be used as a conversational resource and what sorts of video mediation can support full gaze awareness in that role (Gale 1998; Ishii, Kobayashi, \& Grudin, 1993). An obvious preliminary to that research is to determine how accurately people can determine what someone is looking at under natural and video-mediated conditions.

\section{PREVIOUS STUDIES OF THE ACCURACY WITH WHICH GAZE DIRECTION CAN BE ESTIMATED}

Gibson and Pick (1963) performed an experiment in which a gazer looked steadily toward an estimator, who had to judge whether the gazer was looking either at the bridge of his or her nose or elsewhere. There were, in fact, seven gaze targets arranged horizontally around the central target, and the distribution of yes responses was plotted for each position. Gibson and Pick were interested in describing the proximal stimulus for this task - that is, a description of the stimulus for the estimator that captures the ecological significance of the behavior. For this reason, the distribution of yes responses is described in terms of the rotation of the gazer's eyes (his or her head was constrained to face the estimator by a chinrest). People are extremely accurate at this task. The standard deviation of the distribution is $2.8^{\circ}$ of eye rotation, which they compute as representing less than $1 \mathrm{~mm}$ of frontal displacement. Displacing the gazer's head by rotating the chinrest so that his or her head was facing $30^{\circ}$ to the right or the left had only small effects, producing a slight bias in the estimates of $3^{\circ}$ in the direction the head was facing.

A modification to this study allowed the estimator more responses than simply yes and no. Cline (1967) had 13 targets, 3 displaced different amounts upward from the central position, 3 downward, 3 rightward, and 3 leftward. The responses were made via a transparent response board containing 65 positions. This changes the task from one of estimating whether someone is looking one in the eyes (mutual gaze) to one of estimating in what direction someone is looking (partial gaze awareness). It is not an estimation of full gaze awareness accuracy, as defined above, since the estimator cannot see the objects at which the gazer is looking. The use of a response board made it possible for Cline to separate vertical and horizontal error. The standard deviation of these errors was computed as a measure of discrimination, and the mean as a measure of bias. Both measures were notably smaller for the central versus the displaced targets. Taking the standard deviations, for example, vertical error represented $1.25^{\circ}$ of gazer eye rotation. The standard deviation for positions vertically or horizontally displaced was around three times this value. Similarly, the standard deviation of horizontal error represented $0.75^{\circ}$ of gazer eye rotation, and again the standard deviations for displaced positions were around three times larger. Cline also varied head position and obtained small effects of bias that were in line with Gibson and Pick's (1963) result.

Even though this bias from head position is small enough not to be of great practical importance, it has received attention by other investigators. Maruyama and Endo (1983) used line drawings of faces, with the eyes being embedded in a circular representation of a face. Anstis, Mayhew, and Morley (1969) looked at bias caused by head rotation as a function of gaze position and of whether the head was viewed as a real three-dimensional object or as a two-dimensional video image. Bias in the direction of head turn, as observed by Gibson and Pick (1963), was exaggerated when the stimulus was a video image. Anstis et al. were interested in this manipulation for theoretical reasons. They hypothesized that it may be less easy to correctly interpret the combined effects of head turn and eye-within-head rotation when the stimulus is a flat video image, rather than a real three-dimensional head. More recent studies have only examined people's ability to detect when someone is looking them "in the eye"- that is, our third category of gaze awareness, mutual gaze (Argyle \& Dean, 1965; Martin \& Jones, 1982; Martin \& Rovira, 1981; Patterson, 1975; Vine, 1971; von Cranach \& Ellgring, 1973).

To summarize, three kinds of gaze awareness have been distinguished here: full gaze awareness (knowledge of what someone is looking at), partial gaze awareness (knowledge of the general direction someone is looking in), and mutual gaze. Most previous work has been concerned with the latter category. Full gaze awareness is potentially valuable as a conversational resource, since it makes it possible to monitor the other person's focus of visual attention, yet the accuracy with which people can estimate gaze focus has not been measured. Cline's (1967) experiments provide some evidence that such estimations may be rather accurate, but they do not answer the practical question set out here, because the locations the gazer looks at are not visible to the estimator.

\section{GENERAL METHOD}

The two experiments described in this paper measure the accuracy with which full gaze awareness can be achieved under face-toface and video-mediated conditions. A trial in each of these experiments involved a gazer looking at some point also visible to an estimator. The points were labeled and the estimator's task was simply to name that point. The work context to which these results are intended eventually to be applied is one in which two people are conversing about some objects they both can see. Imagine two people sitting either side of a table with some objects on it between them. The estimator is guessing what object the gazer is looking at. In such a situation, the stimulus used by the estimator to achieve this judgment is the position of the gazer's head and eyes, in relation to the objects between them. If the objects are all on a single plane, this stimulus can be described simply in terms of the head-and-eye rotation required for the gazer to look at the object, relative to some 
arbitrary direction, such as straight ahead. In this paper, this headand-eye rotation is decomposed into an up-down component (tilt) and a left-right component (pan). Straight ahead is defined as a point directly in front of and at the same height as the gazer's eyes.

In the first experiment, the errors in the estimation of pan and of tilt were examined separately. For the tilt data, the gazer looked at marks on a line drawn from just below the gazer's head toward the estimator. Because the gazer is looking down on this flat stimulus, a greater head-and-eye rotation is required to move the same linear distance when that distance is nearer, rather than farther. Thus, the marks most distant from the gazer on the scale were more widely spaced than the nearer ones. The distances between points were computed to induce a $2^{\circ}$ change in head-and-eye tilt when the gaze focus of the gazer moved between two adjacent marks, wherever they were on the line. For the pan data, the gazer looked at a semicircular arc, centered on a point immediately below the gazer's eyes. Again, the scale was marked so that a shift of gaze focus between adjacent marks required a $2^{\circ}$ head-and-eye rotation. In the second experiment, the gazer looked at a grid formed by a series of arcs divided into segments. Here, moving between two points on the grid could require a combination of the pan and tilt components of headand-eye rotations. Again, the points were arranged so that moving between adjacent points implied a constant unit of head-and-eye rotation, wherever they were on the grid.

Previous experiments in which the accuracy of gaze awareness has been assessed have typically constrained the position of the head with a chinrest. This would seem to be very artificial. In our experimental task, the gazer looks down onto a position between the gazer and the estimator. In this more realistic situation, the gazer's eyes are not always entirely visible, and head position may be the major stimulus for the estimator. Interestingly, Cline's (1967) Experiment 3 looked at horizontal error when the head was allowed to follow the eyes. Error, as assessed by the standard deviation of estimated position, was then no longer a function of position. The targets displaced horizontally from mutual gaze were as accurately identified as the central one, suggesting strongly that artificially constraining the head position led to much of the error observed in these studies. For these two reasons, gazers in both experiments were instructed to gaze "naturally," and no constraints were put on head movements.

\section{EXPERIMENT 1}

A previous experiment, not reported here, showed that gaze estimation was very accurate. Two possible ways that the estimator might be achieving this accuracy suggest themselves. Our procedure involves the gazer's looking at three calibration points before gazing at the target position. One possibility is that the estimator follows the gazer's head-and-eye movement from the last calibration point. This will be described as movement monitoring. The alternative explanation is that the estimator maps the perceived head-and-eye position of the gazer on to some internal representation of the head-and-eye positions assumed when he or she gazed at the different points. This will be described as position mapping. If the former explanation is correct, the estimator would have to actually see the head-and-eye movement in order to be able to make an accurate judgment. In the latter case, he or she would not. This distinction is of some practical importance, given the aim of this research. We wish to demonstrate the feasibility of monitoring gaze focus as a conversational resource. People do not continuously monitor the eye movements of someone they are talking to. Kendon (1967) described how speakers only gaze at listeners for short periods while they are speaking. Watts and Monk (1996) found that pairs conducting a discussion task over a video link spent less than $25 \%$ of the time they were speaking looking toward the video monitor. This means that, in a real conversation, gaze focus can only be estimated by using position mapping, since it is unlikely that they will be looking at the time a shift in gaze focus is made. If our estimators can only perform this task by monitoring eye movements, the high accuracy of gaze estimates observed cannot be generalized to more natural conversational settings.

Thus, one independent variable in this experiment was whether the eye movement between a calibration point and the target point was visible. The other was the mediumthat is, whether the view provided to the estimator was video mediated. In addition, the points gazed at were divided into three ranges, for the purpose of randomizing trials and scoring the results. For tilt, the ranges are described as near, mid, and far; for pan, as right, mid, and left (from the gazer's point of view). This makes it possible to examine the effect of gaze position on gaze estimation accuracy.

\section{Method}

Participants. Three pairs of students from York University were used. The participants in all the pairs knew each other prior to the experiment. One person in each pair was designated the gazer, whereas the other was the estimator, throughout the experiment.

Materials and Apparatus. Both viewing conditions were designed to be optimal in terms of the lighting and equipment used. The gazer and the estimator were seated $175 \mathrm{~cm}$ apart, on heightadjustable chairs. Between the gazer and the estimator was a 70-cmhigh table, $75 \mathrm{~cm}$ wide, upon which the target scale for the gazer was placed. The height of each participant's eyes from the surface of the table was adjusted to be $50 \mathrm{~cm}$. In the video condition, the gazer sat in the same position on one side of the table, while a video camera was placed where the estimator's head would have been. The estimator sat $70 \mathrm{~cm}$ away from a TV monitor, which gave a video image of the gazer and the target scale. It was computed that this distance would give a stimulus that had the same visual angle as that in the face-to-face condition.

Two white boards measuring $76 \times 104 \mathrm{~cm}$ were used as targets for the gazer. On one a horizontal arc was drawn, so that any point on the arc was $50 \mathrm{~cm}$ from the gazer. Forty-eight marks were made, one every $1.74 \mathrm{~cm}$, and were labeled with numbers. This means that the gazer had to make a head-and-eye panning rotation of $2^{\circ}$ to change gaze focus between adjacent marks. The midpoint of the arc was also marked. The extreme marks on this scale required $94^{\circ}$ of pan rotation each side of this midpoint. This is termed the pan scale.

The other white board contained a line stretching toward the estimator, with markings every $2^{\circ}$ of head-and-eye tilt. These were not equidistant; rather, they were computed in such a way as to require a $2^{\circ}$ change in the tilt of the gazer's head and eyes when moving between adjacent points. Eighteen marks were made and numbered. Again, the midpoint of the line was also marked. The furthest mark from the gazer required a head-and-eye tilt from horizontal straight ahead of $29^{\circ}$, the mid point $46^{\circ}$, and the nearest point $63^{\circ}$, a total of $38^{\circ}$ for the whole scale. This stimulus was termed the tilt scale. For the purpose of randomization and scoring, the points on both scales were divided into three equal ranges. 

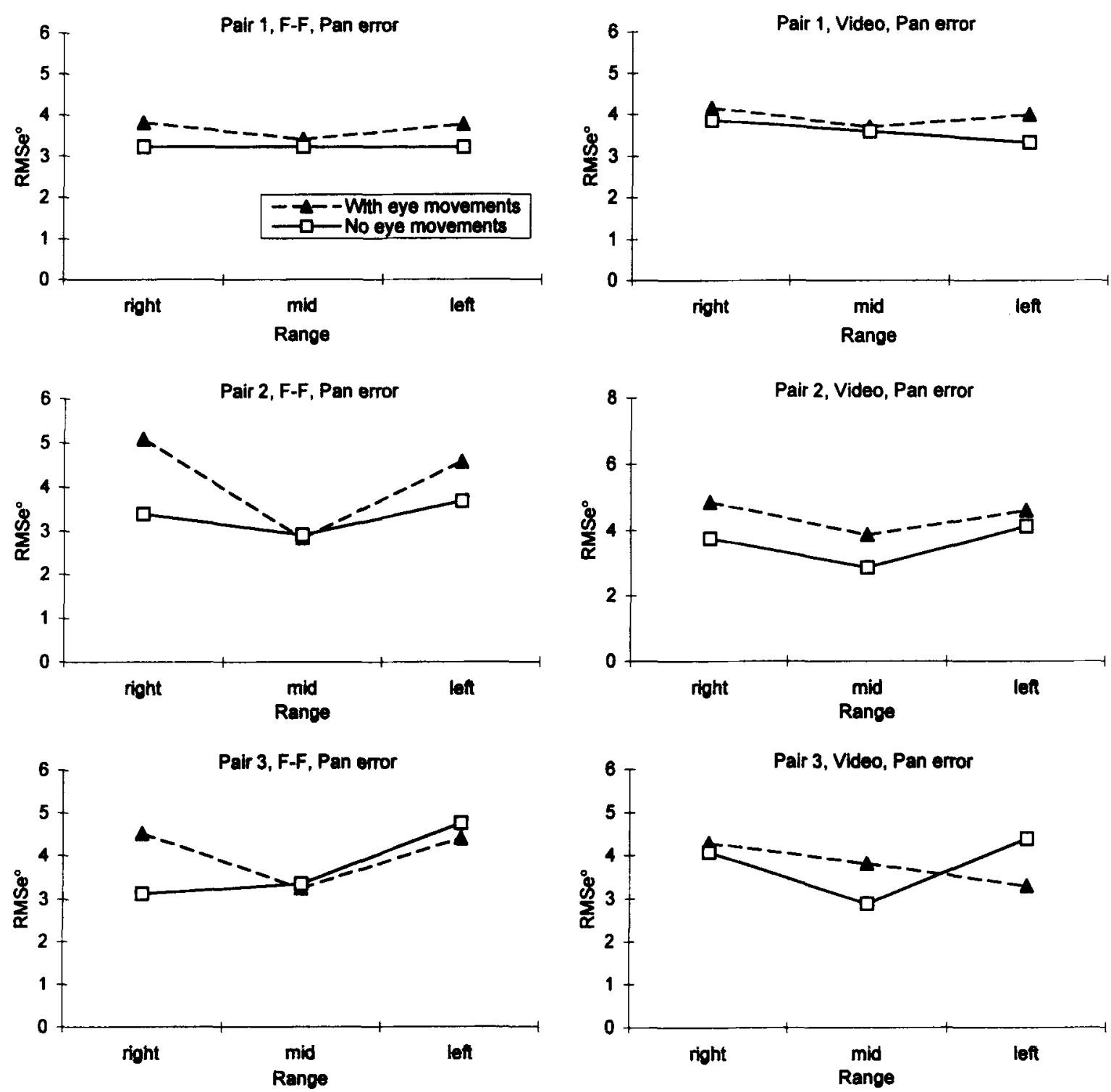

Figure 1. Pan data for individual pairs in Experiment 1. Root mean square error in degrees $\left(R M S_{e}^{\circ}\right)$ is plotted against range for each condition, averaging over session. F-F refers to the face-to-face condition. The ranges (right, mid, and left) refer to the gazer's point of view.

Procedure. The participants were told that this was "an experiment to determine how accurately one person can tell where another person is looking." In all the conditions, the gazer was told to focus on each of the extreme points on the scale, then on the midpoint, before gazing at the designated mark. This was to serve as a calibration procedure for the estimator. In the conditions in which the head-and-eye movements were not visible, the estimator closed his or her eyes between the gazer's looking at the last calibration point and focusing on the designated scale point. After the estimator had made his or her guess, the gazer gave feedback by telling him or her whether the guess was correct or incorrect and, if incorrect, what the correct point was.

Design. Each of the eight experimental sessions consisted of eight conditions, with 15 trials per condition (i.e., 120 trials per ses- sion). The conditions were the eight possible combinations of medium, scale, and visibility of head-and-eye movements. The order in which the conditions were presented was varied, using a Latin square. The order in which the 15 scale points in each condition were presented was also randomized, using a Latin square to randomize the three ranges. Scale points were then selected at random from within the ranges. No point was used more than once in a session. Each experimental session lasted approximately $55 \mathrm{~min}$, and before each new block of 15 trials was presented, there were 5 practice trials, to allow the estimator to adjust to the new condition. The full design of this experiment is, thus, a five-way within-subjects factorial consisting of session (eight levels) $\times$ medium (face-to-face or video) $\times$ scale $($ pan or tilt) $\times$ eye movements (seen or not seen) $\times$ range (three levels). 

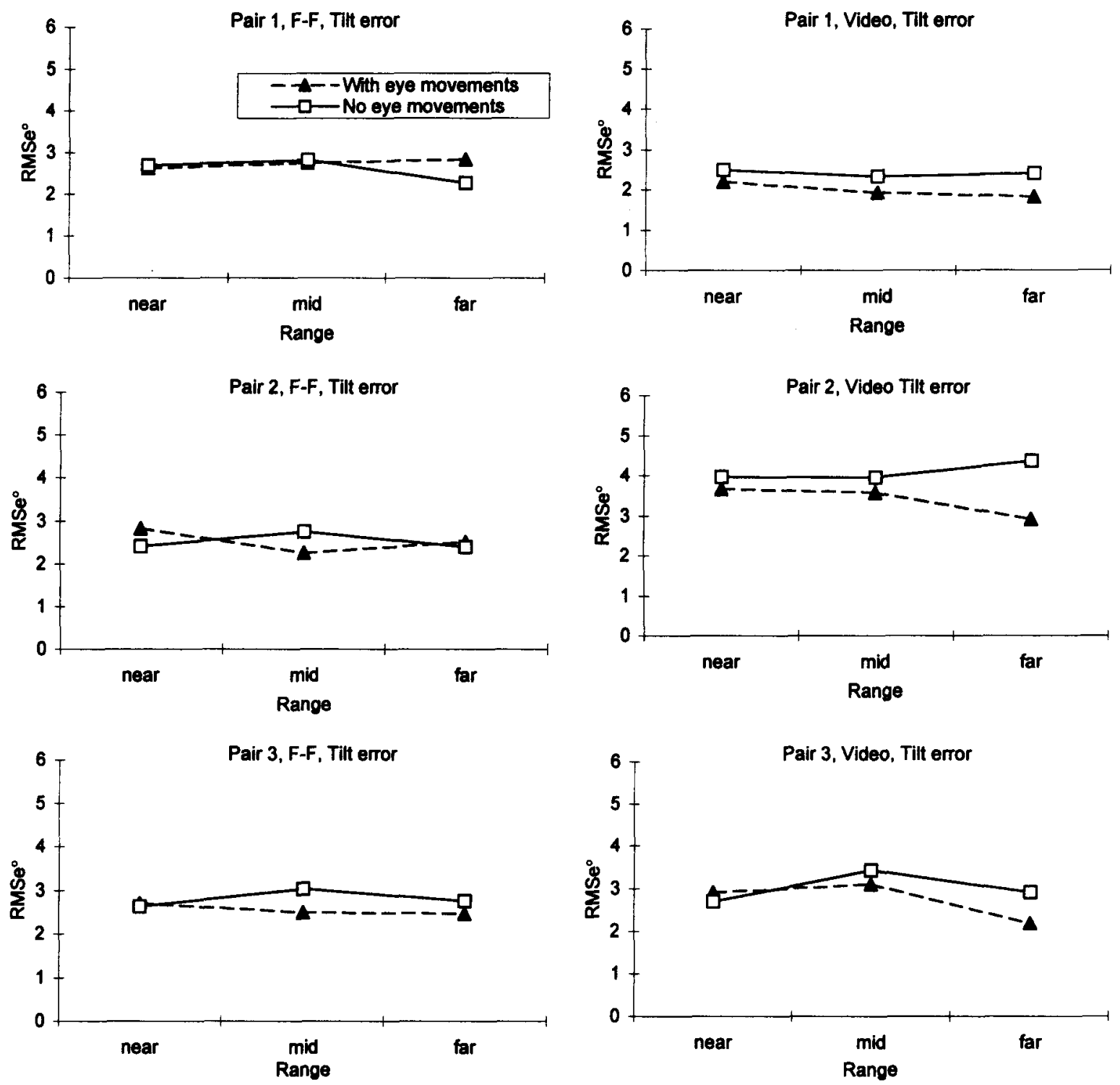

Figure 2. Tilt data for individual pairs in Experiment 1. Root mean square error in degrees $\left(R M S_{e}^{\circ}\right)$ is plotted against range for each condition, averaging over session. F-F refers to the face-to-face condition. The ranges (near, mid, and far) refer to the gazer's point of view.

\section{Results}

All the results in this paper are reported in terms of root mean square error in degrees $\left(R M S_{\mathrm{e}}{ }^{\circ}\right)$. The root of the mean square deviation for the five trials in each treatment combination was determined for use in all subsequent comparisons. In practical terms, an $R M S_{\mathrm{e}}{ }^{\circ}$ of $2^{\circ}$ corresponds to an average error of 1 scale point-for example, choosing mark 25 when the gazer was looking at mark 26 , or vice versa.

Figures 1 and 2 present these data for the three pairs, averaging over sessions. Table 1 presents some means, averaging over pairs. The overall estimates were very accurate. The overall mean $R M S_{\mathrm{e}}{ }^{\circ}$ was $3.84^{\circ}$ for pan and $2.59^{\circ}$ for tilt. Whether the head-and-eye movement to the target was visible (dotted lines in Figures 1 and 2) or not (solid lines) has no discernible effect. Looking at the pan estimates shown in Figure 1, it can be seen that the accuracy of estimation in the three ranges left, mid, and right was very similar - that is, the error of estimation for a particular target was not dependent on the extent of head-andeye rotation. Essentially flat functions were observed in all the cases. The same was true for the tilt estimates shown in Figure 2.

A five-way factorial analysis of variance (ANOVA) was carried out on these data. The five factors were session (eight sessions), medium (video or face-to-face), scale (pan or tilt), range (three levels), and visibility of eye/head movements (visible or not visible). There was a main ef- 
Table 1

Mean Root Mean Square Error in Degrees Averaging

Over Range, Sessions, and Pairs, for Experiments 1 and 2

\begin{tabular}{lcccc}
\hline \multicolumn{1}{c}{ Condition } & F-F Pan & Video Pan & F-F Tilt & Video Tilt \\
\hline $\begin{array}{l}\text { Experiment 1 } \\
\quad \text { Head-and-eye movements visible }\end{array}$ & 3.60 & 4.25 & 2.16 & 2.52 \\
$\quad$ No head-and-eye movements visible & 3.93 & 3.59 & 2.76 & 2.92 \\
Experiment 2 & & & & \\
$\quad$ No head-and-eye movements visible & 2.67 & 3.09 & 2.51 & 2.95 \\
\hline Note-F-F and Video are the face-to-face and video-mediated conditions, respectively.
\end{tabular}

fect of scale, showing that the pan estimates gave significantly higher $R M S_{\mathrm{e}}{ }^{\circ} \mathrm{s}$ than did the tilt estimates $[F(1,2)=$ $38.83, p=.025]$. There were no significant main effects for range, medium, or visibility of head-and-eye movements. There was a session main effect $[F(7,14)=3.70$, $p=.018]$ and a session $\times$ visibility $\times$ range interaction $[F(14,28)=2.24, p=.034]$. These do not reflect recognizable trends, such as practice effects; rather, they seem to be due to one or two aberrant points. Although these effects may be statistically significant, they are uninteresting. No other interaction was significant.

In summary, estimates of gaze focus are very accurate, and making these judgments is no more difficult over a video link than when face-to-face over a table in the same room. The other purpose of this experiment was to examine the effect of visibility of head-and-eye movements on this task. There was no advantage to being able to see the gazer's head-and-eye movements. This favors the idea of a mapping mechanism where participants map the perceived head-and-eye position against an internal representation, rather than monitoring the head-and-eye movement. From an ecological viewpoint, this makes sense. As was pointed out above, a gaze awareness mechanism reliant on monitoring the movement of the gazer would not be robust, since it would depend on the real-world equivalent of our estimator's spending nearly all of his or her time focusing on the gazer. The result is also interesting from the point of view of a designer specifying the video configuration needed to support gaze awareness. It suggests that full-motion video may not be necessarythat is, low refresh rates may provide sufficient information for full gaze awareness.

\section{EXPERIMENT 2}

The essentially flat functions obtained when plotting error of gaze estimation against gaze extent (range) are encouraging with regard to our analysis of the stimulus the estimator was using. The estimator's task was characterized here as one of estimating the angular extent of the gazer's head-and-eye rotation with respect to some standard direction. If the gazer had been using some other cue, the results might not have been so straightforwardly described. However, two elements in the procedure used in Experiment 1 could be argued to have biased the results in this direction. First, only one dimension of head- and-eye rotation was used in a particular trial: Either the estimator was asked to estimate head-and-eye tilt, or he or she was asked to estimate head-and-eye pan. In Experiment 2 , the gazer was given a combined pan-and-tilt grid, so the estimator had simultaneously to estimate pan and tilt rotation. Second, on each trial in Experiment 1, the gazer looked at three standard calibration points before gazing at the target. In Experiment 2, this part of the procedure was dropped, and the gazer simply moved directly from one target to another. There was no condition in which head-and-eye movement was visible in Experiment 2 . The estimator closed his or her eyes while the eye movement was made, as in the condition in which headand-eye movement was not visible in Experiment 1.

\section{Method}

Participants. Six participants were used in three pairs. They consisted of 6 female students at York University. As in Experiment 1,1 subject in each pair was the gazer throughout the experiment, while the other was the estimator.

Materials and Apparatus. One white board measuring $76 \times$ $104 \mathrm{~cm}$ was used. On it, a combined pan-and-tilt grid of eight columns and six rows was drawn. Each of the rows was a colored horizontal arc, so that any point on the individual row was the same distance from the gazer. Each row had eight equidistant marks on it, numbered from 1 to 8 . Each of the 48 points in the array was, therefore, labeled by a color (tilt) and a number (pan). Each point in the array required the gazer to rotate her head $4^{\circ}$ to move from the immediately surrounding points. Moving from top to bottom of any column in the grid required a $24^{\circ}$ tilt rotation and no pan rotation. Moving from left to right along any row (arc) required a $32^{\circ}$ pan rotation and no tilt rotation. It was decided to make each point $4^{\circ}$ rather than $2^{\circ}$ apart, since pilot experiments had indicated that judgments may be more difficult to make with a two-dimensional grid than with the one-dimensional scale used in Experiment 1.

Design and Procedure. In order to speed up the process of generating and recording data, the locations to be gazed at were communicated over an audio link. The gazer wore headphones, so that the estimator could not overhear the experimenter reading out these locations. In addition, in the video condition the estimator wore a combined headphone and microphone set that allowed two-way communication with the gazer. The link between the gazer and the experimenter was two-way, whereas the link between the estimator and the experimenter was one-way (i.e., the experimenter could hear what the estimator was saying, but not vice versa). The experimenter relayed a list of grid points to the gazer, one point at a time, over the audio link. The estimator closed her eyes while the gazer searched the array for the specified point. Once the gazer's gaze was focused, the estimator opened her eyes and guessed at which point the gazer was looking. The gazer then gave feedback, and the guess was recorded by the experimenter. 

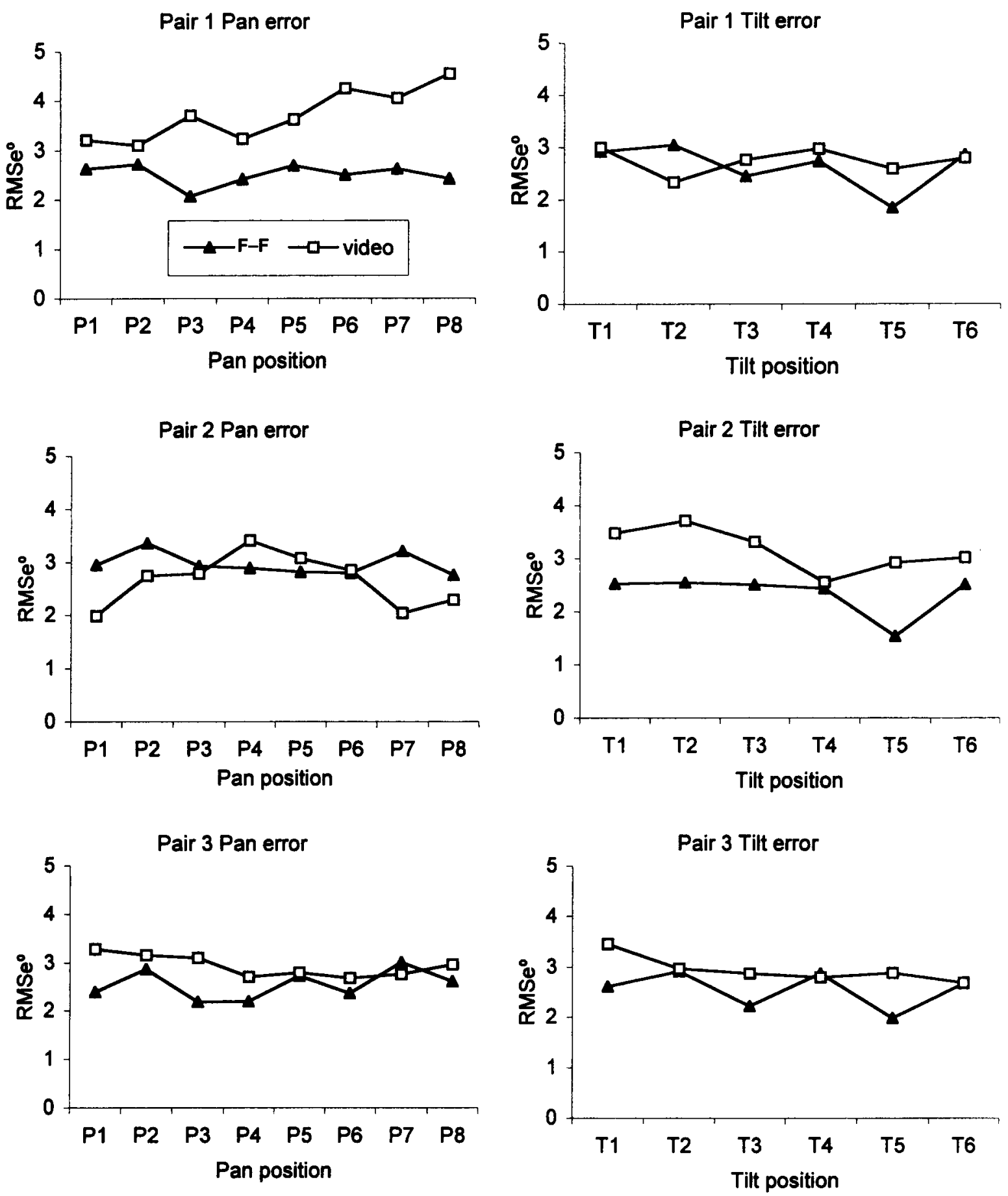

Figure 3. Data for individual pairs in Experiment 2. Pan error (given as root mean square error in degrees $\left[R M S_{e}^{\circ}\right]$ ) is plotted against pan position, and tilt error is plotted against tilt position for each medium, averaging over sessions. Straight ahead lies between p4 and p5; $t 1$ (near) is further from straight ahead than t6 (far). F-F refers to the face-to-face condition.

Each experimental session consisted of two blocks of trials, the blocks being the two possible conditions of face-to-face and video. Each grid point was gazed at twice per condition per session and the order in which the points were presented was randomized. Given that the grid contained 48 points, this meant 192 trials per session, plus 10 practice trials at the beginning of each new condition. There were five sessions overall- that is, each point was gazed at 10 times per condition. The design of this experiment was thus a four-way within-subjects factorial consisting of session (five levels) $\times$ medium (face-to-face or video) $\times$ pan (eight levels) $\times$ tilt (six levels) .

\section{Results}

Experiment 2 differed from the previous experiment in that each point gazed at varied in the extent of both pan and tilt and the estimator's guess could be in error with re- 
Pair 1 Pan error

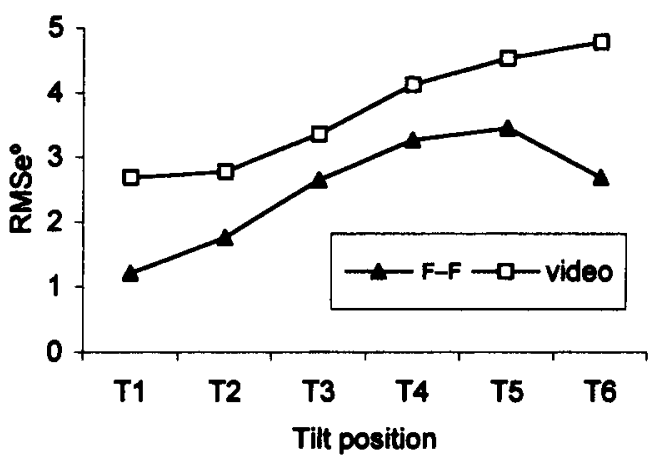

Pair 2 Pan error

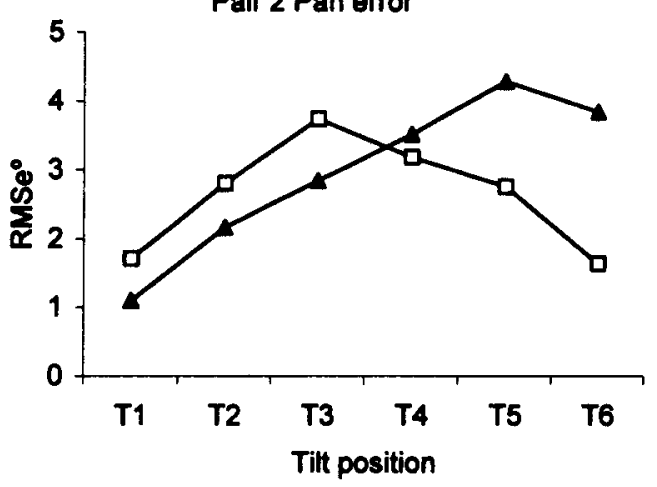

Pair 3 Pan error

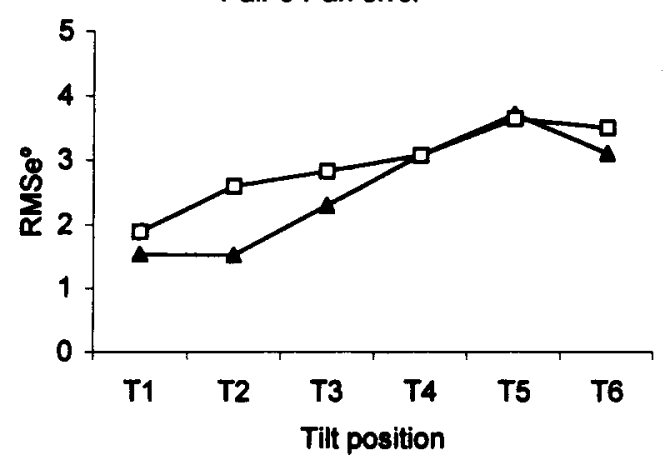

Pair 1 Tilt error
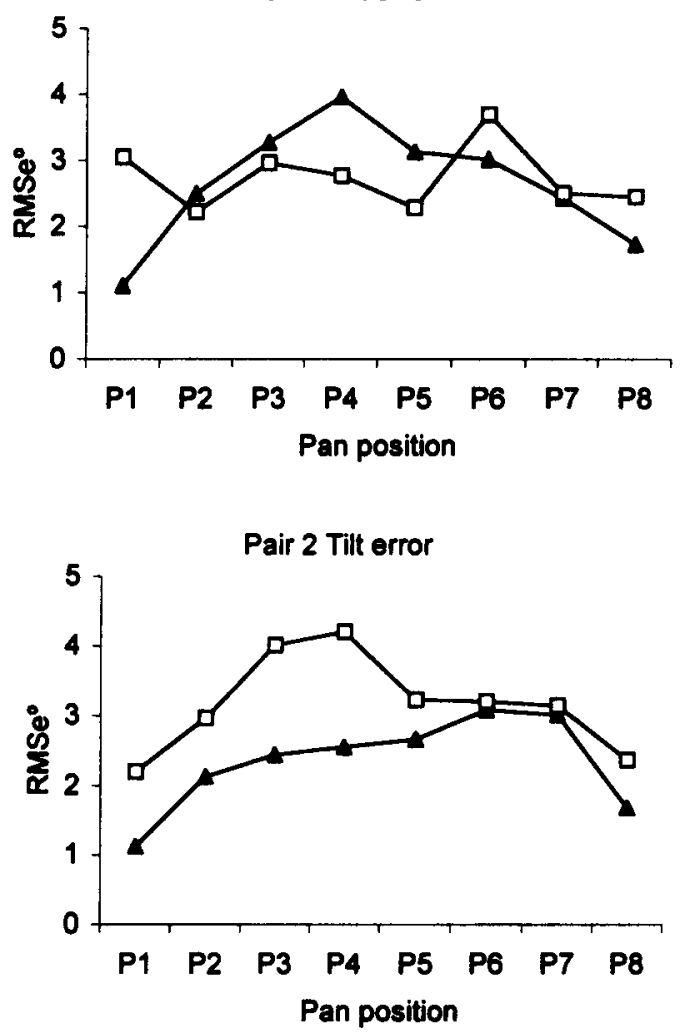

Pair 3 Tilt error

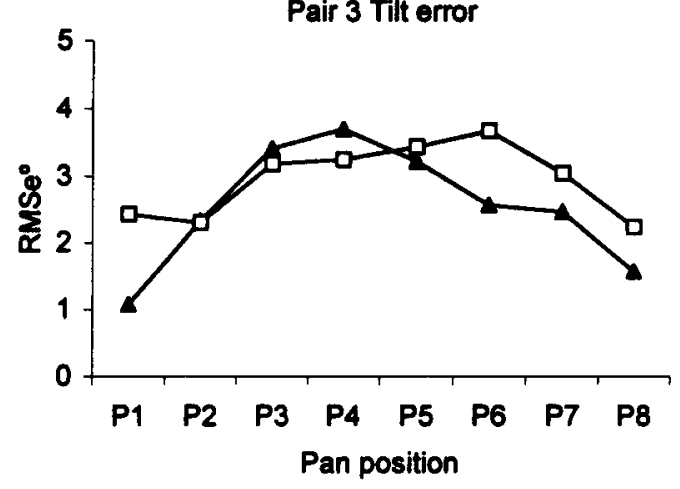

Figure 4. Data for individual pairs in Experiment 2. Pan error (given as root mean square error in degrees $\left.\left[R M S_{\mathrm{e}}{ }^{\circ}\right]\right)$ is plotted against tilt position, and tilt error is plotted against pan position for each medium, averaging over sessions. F-F refers to the face-to-face condition.

spect to both. Root mean square error in the pan dimension was computed separately from root mean square error in the tilt dimension, to permit comparison with Experiment 1 . Figure 3 plots pan error as a function of pan position and tilt error as a function of tilt position and so presents data broadly equivalent to those obtained in Experiment 1 . Figure 4 plots pan error as a function of tilt position and tilt error as a function of pan position. These data were not available from the unidimensional scale used in Experiment 1.
The $R M S_{\mathrm{e}}{ }^{\circ} \mathrm{s}$ in Figures 3 and 4 are very comparable with previous results in showing a high degree of accuracy in the estimates of gaze position. Pan error, averaging over pan and tilt positions and session was $2.67^{\circ}$ $R M S_{\mathrm{e}}{ }^{\circ}$ in the face-to-face condition, and tilt error was $3.09^{\circ}$ $R M S_{\mathrm{e}}^{\circ}$. Table 1 includes further summaries.

In the previous experiment, the degree of error in estimating pan was greater than the degree of error in estimating tilt. In this experiment, in which estimates are no longer constrained to separate dimensions the $R M S_{\mathrm{e}}{ }^{\circ} \mathrm{S}$ 

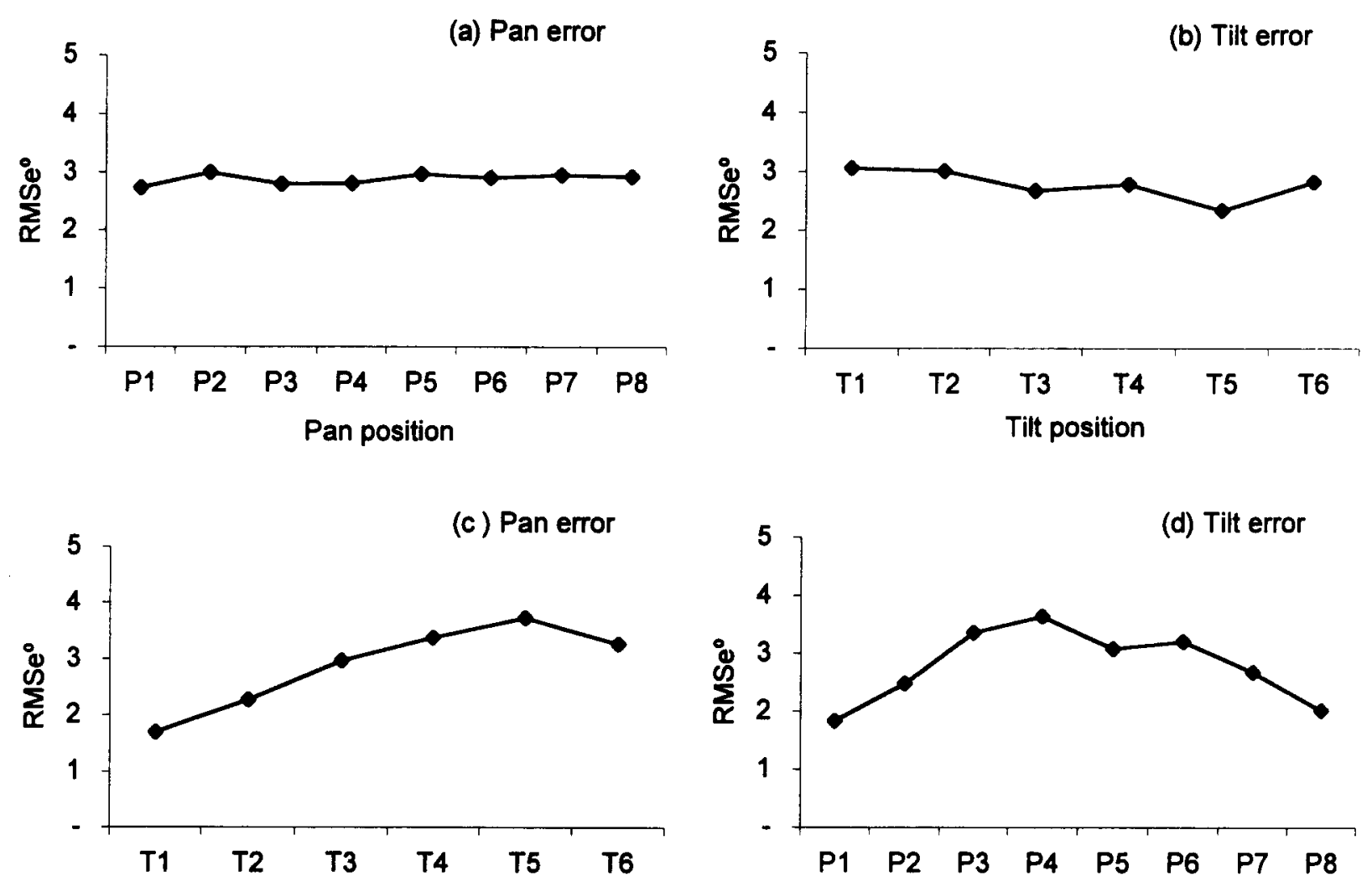

Tilt position

Pan position

Figure 5. Root mean square error in degrees $\left(\boldsymbol{R} M S_{\mathrm{e}}^{\circ}\right)$, averaging over pair, session, and mediation condition, in Experiment 2. Panel a, pan error as a function of pan position; panel $b$, tilt error as a function of tilt position; panel c, pan error as a function of tilt position; panel d, tilt error as a function of pan position.

are much more comparable. There also seems to be some small (less than $0.5^{\circ}$ of $R M S_{\mathrm{e}}^{\circ}$ ) disadvantage in the video condition, as compared with face-to-face estimates.

Four two-way factorial repeated measures ANOVAs were carried out on the basis of the data plotted in Figures 3 and 4, with medium (face-to-face or video) and either pan position (eight levels) or tilt position (six levels) as factors. What significant effects there were were all main effects of pan or tilt position; there were no main effects of medium and no two-way interactions with medium. An analysis of pan $R M S_{\mathrm{e}}^{\mathrm{o}}$, with medium and pan position as factors, showed no significant effects. A plot of pan $R M S_{\mathrm{e}}{ }^{\circ}$ against pan position was essentially flat, as in Experiment 1 (see Figure 5a). An analysis of tilt $R M S_{\mathrm{e}}{ }^{\circ}$, with medium and tilt position as factors, showed a main effect of tilt position $[F(5,10)=6.35, p=.007]$. Errors of estimation in tilt decreased slightly from tilt positions $\mathrm{T} 1$ to $\mathrm{T} 5$ - that is, as the tilt position got nearer to straight ahead; however, this decrease was small (see Figure $5 \mathrm{~b}$ ). An analysis of pan $R M S_{\mathrm{e}}{ }^{\circ}$, with medium and tilt position as factors, showed a main effect of tilt position $[F(5,10)=21.11, p<.001]$. Errors of pan estimation increased as tilt position got nearer to straight ahead (see Figure $5 \mathrm{c}$ ). Finally, an analysis of tilt $R M S_{\mathrm{e}}{ }^{\circ}$, with medium and pan position as factors, showed a signifi- cant main effect of pan position $[F(7,14)=25.76, p<$ $.001]$. Errors of estimation in tilt increased with pan positions nearer to straight ahead (see Figure $5 \mathrm{~d}$ ).

In conclusion, the most striking finding was again the high levels of accuracy across all conditions: $R M S_{\mathrm{e}}{ }^{\circ}$ was less than $5^{\circ}$ in the most difficult condition. As was previously stated, it was thought that judgments on a combined pan-and-tilt grid might be more difficult, and thus it was decided to make the distance between grid points $4^{\circ}$, rather than the $2^{\circ}$ used in Experiment 1 . In retrospect, this may have been a mistake, since a distance of $4^{\circ}$ between points resulted in judgments so accurate that a ceiling effect might have been occurring. Although this was unfortunate, in that it may have reduced the effects of the independent variables used, it did confirm previous findings that people can make these judgments to a high degree of accuracy even over a video link.

\section{CONCLUSIONS}

The purpose of these experiments was to demonstrate that full gaze awareness is possible with sufficient accuracy to be used as a resource in face-to-face and videomediated conversations. Although it may be much harder to monitor gaze focus under the circumstances of normal 
conversation, as compared with the idealized experimental context used here, the results are very encouraging. If we assume symmetrical, normally distributed error and an optimum detection criterion, an $R M S_{\mathrm{e}}$ of $3^{\circ}$ means that a difference in gaze position resulting in $6^{\circ}$ of head-andeye rotation will be correctly detected $84 \%$ of the time and a $12^{\circ}$ head-and-eye rotation $98 \%$ of the time. Furthermore, given the small changes that need to be perceived to detect a $3^{\circ}$ change in the head-and-eye rotation of a gazer at a distance of $175 \mathrm{~cm}$, one might have expected the inevitable degradation of signal imposed by video mediation to have reduced performance. These experiments show that, even within the limitations imposed by domestic video equipment, it is possible to provide a stimulus for the estimator that is as effective as the real thing. It would seem that people can readily distinguish gaze focus with some accuracy. The next step in this research will be to show that people actually use this information in conversation. The aim would be to demonstrate an advantage for video configurations that include a single view of the face and the objects being looked at, rather than a view of the face or a view of the objects on their own (Gale, 1998).

This research has a practical motivation. However, as is generally the case with applied research, the results of these experiments are also of theoretical interest. The essentially flat functions obtained in Experiment 1 when plotting accuracy against pan and tilt extent (range) were interpreted as supporting our analysis that the estimator's task was one of estimating head-and-eye rotation, rather than some other stimulus attribute. In Experiment 2, pan position affected tilt error but not pan error. One might have expected some advantage for the straight ahead position, since here the eyes can be seen most clearly, but this was not apparent in the data. Indeed, tilt error was worst for this position. One may speculate that the judgment is made mainly on the basis of head position and that a half profile gives more tilt information than does a full-face view. The effect of tilt position on tilt error was small and can probably be discounted, but the effect of tilt position on pan error was marked and is most puzzling. Near-tilt positions, when the eyes were most obscured by the eyelids and eyebrows, resulted in less error than did fartilt positions, when the face was lifted more toward the estimator.

The high accuracy of estimation observed in all these data is also of theoretical interest. The estimators were able to achieve this level of performance with very little practice and showed no perceptible learning throughout the experiments, suggesting that the judgments required were natural and well practiced when the experiment began. Thus, it would seem that knowledge of what someone else is looking at is used habitually in everyday life. The estimators were still very accurate when they could not see the head-and-eye movement to the gazed-at object. This result rules out models of how such a judgment is made that depend on the monitoring of that movement.

\section{REFERENCES}

Anderson, A. H., O’Malley, C., Doherty-Sneddon, G., Langton, S. Newlands, A., Mullin, J., Fleming, A. M., \& van der Velden, J (1997). The impact of VMC on collaborative problem solving: An analysis of task performance, communicative process, and user satisfaction. In K. E. Finn, A. J. Sellen, \& S. B. Wilbur (Eds.), Videomediated communication (pp. 133-155). Mahwah, NJ: Erlbaum.

Anstis, S. M., Mayhew, J. W., \& Morley, T. (1969). The perception of where a face or television 'portrait' is looking. American Journal of Psychology, 82, 474-489.

ARGYLE, M., \& DeAN, J. (1965). Eye-contact, distance and affiliation. Sociometry, 28, 289-304.

BUXTON, W. A. S., \& MORAN, T. (1990). EuroPARC's integrated interactive intermedia facility (iiif): Early experience. In S. Gibbs \& A. A. Verrijn-Stuart (Eds.), Multi-user interfaces and applications (pp. 1134). Amsterdam: Elsevier.

CLARK, H. H. (1996). Using language. Cambridge: Cambridge University Press.

Cline, M. G. (1967). The perception of where a person is looking. American Journal of Psychology, 80, 41-50.

GALE, C. (1998). The effects of gaze awareness on dialogue in a videobased manipulative task. In C. Karat \& A. Lund (Eds.), CHI'98 (pp. 345-346). New York: ACM Press.

GaVer, W., Smets, G., \& Overbeeke, K. (1995). A virtual window on media space. In I. R. Katz, R. Mack, L. Marks, M. Rosson, \& J. Nielsen (Eds.), CHI'95 (pp. 257-264). New York: ACM Press.

Gibson, J. J., \& Pick, A. D. (1963). Perception of another person's looking behavior. American Journal of Psychology, 76, 86-94.

GooDwIN, C. (1981). Conversational organisation: Interaction between speakers and hearers. London: Academic Press.

IshII, H., Kobayashi, M., \& GRudin, J. (1993). Integration of interpersonal space and shared workspace: ClearBoard design and experiments. ACM Transactions on Information Systems, 11, 349-375.

KENDON, A. (1967). Some function of gaze direction in social interaction. Acta Psychologica, 26, 22-63.

MARTin, M., \& Jones, R. F. (1982). The accuracy of eye-gaze judgement: A signal detection approach. British Journal of Social Psychology, 21, 293-299.

Martin, W., \& Rovira, M. (1981). An experimental analysis of discriminability and bias in eye-gaze judgement. Journal of Nonverbal Behavior, 5, 155-163.

Maruyama, K., \& Endo, M. (1983). The effect of face orientation upon apparent direction of gaze. Tohoku Psychologica Folia, 42, 126-138.

Nardi, B. A., SchWarz, K., KuChINSKY, A., LeIChNER, R., WhitTAKER, S., \& SCLABASSI, R. (1993). Turning away from talking heads: The use of video-as-data in neurosurgery. In S. Ashlund, K. Mullet, A. Henderson, E. Hollnagel, \& T. White (Eds.), CHI'93 (pp. 327334). New York: ACM Press.

Patterson, M. L. (1975). Eye contact and distance: A re-examination of measurement problems. Personality \& Social Psychology Bulletin, $1,600-603$.

Siegel, J., Kraut, R. E., \& Miller, M. D. (1996). Turning the camera around: Collaboration and video communication in performance of physical tasks. In M. J. Tauber (Ed.), CHI'96 (pp. 113-114). New York: ACM Press.

VINE, I. (1971). Judgement of direction of gaze: An interpretation of discrepant results. British Journal of Social \& Clinical Psychology, 10, 320-331.

von Cranach, M., \& Ellgring, J. H. (Ed.) (1973). Problems in the recognition of gaze direction. London: Academic Press.

WATTS, L. A., \& MonK, A. F. (1996). Remote assistance: A view of the work AND a view of the face? In M. J. Tauber (Ed.), CHI'96 (pp. 101-102). New York: ACM Press.

(Manuscript received May 27, 1998 revision accepted for publication January 11,1999 .) 